\title{
Treatment Site Reactions to Zyderm Collagen Implantation
}

\author{
NEIL A. SWANSON, M.D. \\ JOHN G. STONER, M.D. \\ RONALD J. SIEGLE, M.D. \\ ALVIN R. SOLOMON, M.D.
}

\begin{abstract}
Two cases of treatment site granulomatous reaction to Zyderm Collagen Implant that occurred in spite of a negative skin test are reported. A delayed hypersensitivity reaction to $\mathrm{ZCI}$ is suggested by the clinical time course and the histologic findings of hypersensitivity granulomas. Humoral immunity is also implicated in the reaction since elevated anti-Zyderm serum antibodies and plasma cell infiltrates are present. Resolution of the clinical signs and symptoms occurred over several months with no treatment. The physician using ZCI should be aware of this rare adverse reaction.
\end{abstract}

Injectable collagen implant (Zyderm) has been promoted for the correction of soft-tissue contour defects. Scars amenable to treatment include certain depressed acne scars, age lines, rhytids, viral pox scars, traumatic scars, iatrogenic scars, and certain postsurgical scars. Zyderm Collagen Implant $(\mathrm{ZCl})$ is purified bovine dermal collagen which has undergone selective proteolytic hydrolysis of its telopeptide portions to decrease its immunogenicity. It is suspended in a physiological saline buffer containing $0.3 \%$ lidocaine for injection. After intradermal injection, the substance becomes incorporated into the host's dermal collagen matrix. Patients are given a skin test with ZCI before therapeutic use to detect potential sensitivity to the implant. A positive reaction has been reported in about $3 \%$ of patients. ${ }^{1}$ Histologically, positive skin tests show a foreign-body-like

Drs. Swanson, Stoner, Siegle, and Solomon are with the Department of Dermatology, and Dr. Solomon is also affiliated with the Department of Pathology, University of Michigan Medical School.

Address reprint requests to Neil A. Swanson, M.D., University of Michigan, Department of Dermatology, C2065 Outpatient Building, Ann Arbor, MI 48109. granuloma. In the presence of a negative skin test, adverse reactions in treatment sites have not been reported. This paper presents two patients with such reactions and defines their clinical course.

\section{CASE REPORTS}

\section{Case 1}

A 36-year-old white woman presented for treatment of severe acne scarring. After discussion of treatment options, $\mathrm{ZCI}$ followed by dermabrasion was chosen. A 0.1-cc test dose was implanted in the right forearm in the usual fashion. The patient had no reaction at 48 hours and at 1 month the test site was clinically and histologically negative. Two cc of $\mathrm{Zy}$ derm were implanted in three areas of the right cheek. Eighteen days postinjection, the patient developed persistent swelling and firmness in the area. She presented, 21 days postinjection, with induration and an urticarial violaceous plaque at the injection site as well as with right periorbital edema. The test injection site on her forearm remained negative. She denied systemic symptoms. Prednisone, $40 \mathrm{mg} /$ day for 3 days, was given for the periorbital edema which then rapidly subsided. The indurated and violaceous nodular lesions at the injection sites persisted (Fig. 1). Because of the patient's history of chronic sinus infections and the reported sinus problems concurrent with ZCI allergy, she was begun on cephadroxil 500 $\mathrm{mg}$ daily. This had no effect on the reaction. A biopsy specimen taken from the involved site showed a granulomatous reaction. The serum showed the presence of anti-Zyderm antibodies as measured by the Collagen Corporation, but the patient's antinuclear 


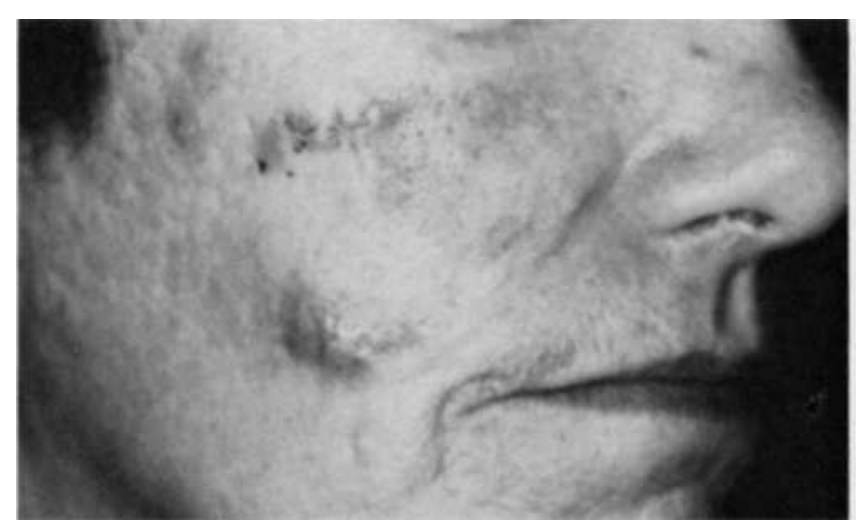

FIGURE 1. Treatment site reactions consisting of erythematous, indurated nodules at sites of injection with Zyderm Collagen Implant.

antibody assay, erythrocyte sedimentation rate, and complete blood count were normal.

The induration and discoloration at the treatment sites on her face gradually resolved over the next 6 months (Fig. 2). During this period, she did occasionally experience a flare-up on her face as well as on the test dose site, manifested by induration and tenderness. This often followed sun exposure.

Case 2

A 42-year-old healthy white woman presented for treatment of two traumatic scars on the left cheek. She had no contraindications to ZCI therapy, and her scars appeared amenable to this therapy. A standard $0.1 \mathrm{cc}$ test dose of $\mathrm{ZCI}$ was implanted in the right forearm. There were no adverse reactions at 48 hours or at 1 month. Clinically her test dose was negative, and treatment was initiated with $0.4 \mathrm{cc}$ of $\mathrm{ZCI}$ in the left cheek. An immediate $130 \%$ correction was achieved, and there was no complication at the time of injection. During the next 2 months, she experienced intermittent redness and induration at the injection site. The test site on her arm remained negative. She denied any systemic symptoms. A biopsy specimen from the left cheek site showed a granulomatous inflammatory reaction. Serum drawn at this time revealed the presence of anti-Zyderm antibodies, but the antinuclear antibody assay and the erythrocyte sedimentation rate were normal. Observation of the lesion over the next several months showed a gradual and complete clinical remission without treatment.

\section{HISTOPATHOLOGIC FINDINGS}

Both biopsy speicmens were fixed in 10\% buffered formalin for 24 hours and then routinely processed for hematoxylin and eosin staining $(H \& E)$. Periodic

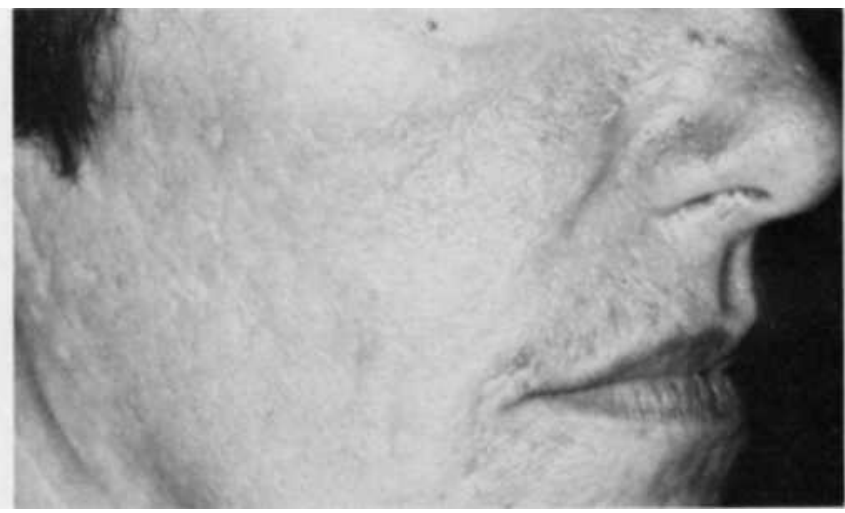

FIGURE 2. Healing of reaction sites at 6 months without treatment.

acid-Schiff (PAS), Movat's pentachrome, and alcian blue (at $\mathrm{pH} 0.4$ and 2.5) stains were also used.

Similar changes were noted in both specimens. The epidermis was unremarkable. Solar elastosis in the papillary and upper reticular dermis was noted. The reticular dermis was extensively altered by a complex admixture of granulomatous inflammation and scar tissue (Fig. 3). These changes expanded the reticular dermis and extended into the subcutis. The cellular constituents of the granulomas ranged from pure epithelioid cells extending radially from a wavy homogeneous eosinophilic central material in a palisaded configuration to relatively loose granulomas composed of the same centrally located homogeneous eosinophilic material surrounded by neutrophils, macrophages, lymphocytes, plasma cells, eosinophils, and occasional foreign body and

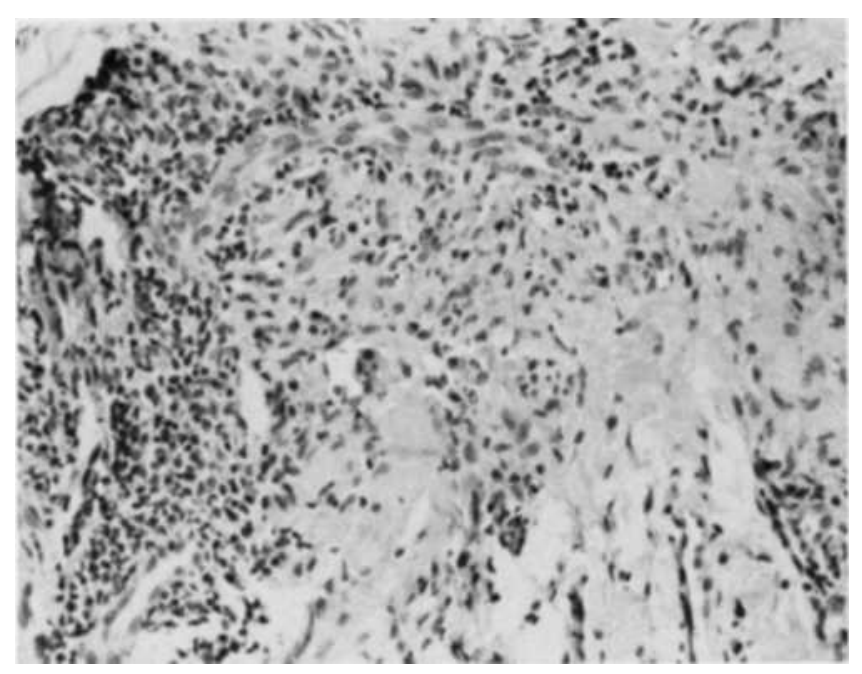

FIGURE 3. A granuloma within dermis is partially surrounded by lymphocytes. 


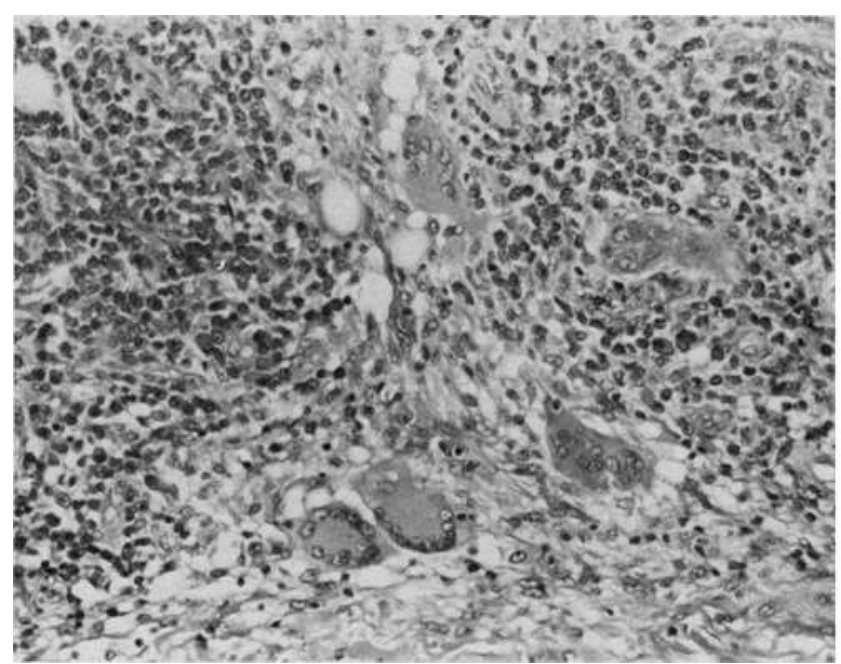

FIGURE 4. Giant cells are particularly prominent in this granuloma.

Langhans' giant cells (Figs. 4 and 5). One granuloma in the deep dermis from Case 2 had a purulent center. Diffuse lymphoplasmocytic infiltrates were scattered throughout the dermis among the granulomas. Focal dense collagenous dermal scar was also present adjacent to the granulomas; no polarizable material was noted in any of the granulomas. The homogeneous material in the center of the granulomas did not stain with PAS or alcian blue stains at either $\mathrm{pH}$. With Movat's stain, considerable loss of elastic tissue was noted in the granulomatous areas. It is assumed that the homogeneous material within the center of some of the granulomas was Zyderm Collagen Implant (Fig. 5).

In conclusion, we present two patients who devel- oped adverse local reactions to ZCI in spite of negative reactions to intradermal skin test at 48 hours and at 1 month. A delayed hypersensitivity-mediated reaction to $\mathrm{ZCI}$ is suggested by the clinical course and the histologic finding of foreign-body-type granulomas within the dermis. The presence of urticaria clinically, elevated anti-Zyderm antibody titers serologically, and plasma cells histologically suggest that humoral immunity is also actively involved in these reactions. Although these reactions were self-limited and local, continued monitoring of adverse reactions to $\mathrm{ZCI}$ is essential to evaluate its ultimate safety in the treatment of dermal defects.

\section{COMMENTS}

More than 10,000 patients have been treated with ZCI since the institution of clinical trials in 1976 and Federal Drug Administration approval for use as a

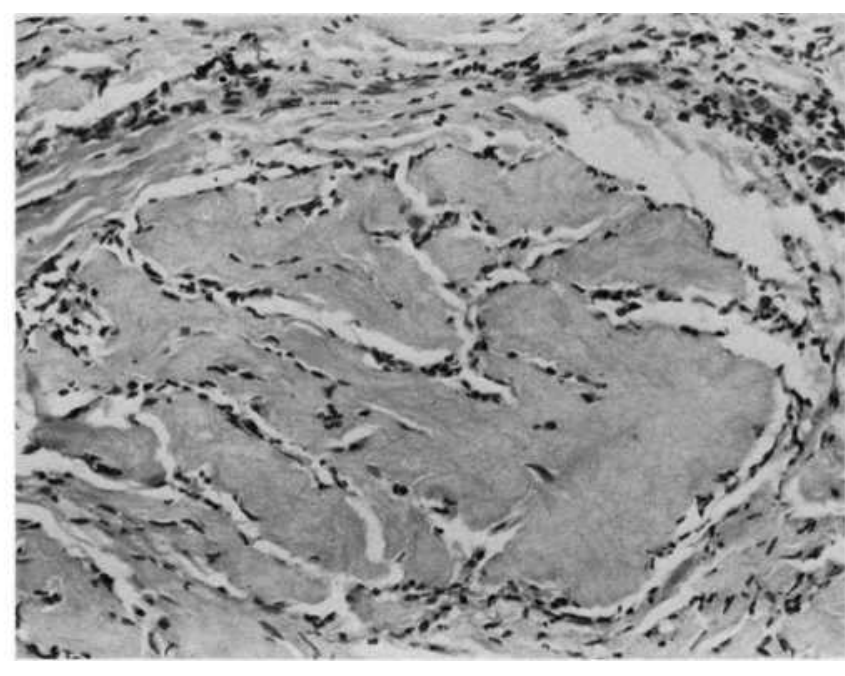

FIGURE 5. A dermal deposit of eosinophilic homogeneous material, presumably Zyderm, shows peripheral rimming and permeation by phagocytes which imparts a convoluted appearance to the deposit.

medical device in August 1981. ${ }^{1}$ We now have treated more than 200 patients with $\mathrm{ZCI}$.

Reported adverse reactions to $\mathrm{ZCI}$ have been rare. Data on file with the Collagen Corporation revealed a positive skin test, described as localized swelling, induration, erythema, pruritus, tenderness, or pain occurring in about $3 \%$ of 9,427 patients. ${ }^{1}$ The reaction usually lasts from 3 days to 3 months. Systemic symptoms of generalized rash, arthralgias, myalgias, generalized pruritus, and mild edema at the injection site have also been reported after skin test implantation. Adverse treatment reactions to injected $\mathrm{ZCI}$ include bruising at the injection site and injection into small facial arteries. Other uncommon adverse responses at treatment sites include infection (viral and bacterial) and transient swelling following alcohol ingestion, sinusitis, or exposure to heat and/or sunlight. These reactions have been reported ${ }^{1,2}$ in 33 of 9,427 patients $(0.4 \%)$.

The histopathology of the granulomatous inflammation at the biopsy site suggests a spectrum ranging from foreign-body granulomas to allergic or hypersensitivity granulomas. Granulomas composed primarily of epithelioid cells that palisade around the central eosinophilic globular mass (Fig. 5) are typical of foreign-body granulomas in which the inciting foreign substance is characterized by low solubility and low toxicity. ${ }^{3,4}$ We assume that the central core of these granulomas is composed primarily of the $\mathrm{ZCI}$ but cannot prove this by light microscopic study. We are presently attempting to develop an immunoperoxidase anti-Zyderm antibody conjugate in order to identify definitively the $Z C I$ in tissue. Other present granulomas contain large numbers of the 
lymphocytes and plasma cells. These are suggestive of hypersensitivity granulomas. ${ }^{+}$The time course of the patient's clinical reaction is certainly consistent with the delayed hypersensitivity reaction. Blast transformation of the patient's lymphocytes in the presence of $\mathrm{ZCI}$ should be assayed to substantiate this supposition. The presence of numerous plasma cells within and adjacent to the granulomas may correlate with the presence of elevated titers of anti-Zyderm antibody in the patient's serum. The wheal and periorbital edema reactions at the injection site may also correlate with the presence of serum anti-Zyderm antibodies since these usually indicate an immediate hypersensitivity reaction mediated by a humoral factor.

Our patients have experienced only local reactions to ZCI. There is no evidence by history or routine serologic data (antinuclear antibody, erythrocyte sedimentation rate) to suggest systemic involvement.
There is no clinical or laboratory evidence of any underlying immunologic abnormality in these patients although they have not been evaluated extensively. However, both patients' serum exhibited anti-Zyderm antibodies when compared to normal controls. The significance of this finding is unknown. The natural course of the reaction seems to be remission over a period of months without development of systemic symptoms.

\section{REFERENCES}

1. Data on file, Collagen Corporation.

2. Stegman, S. J., and Tromovitch, T. A. Implantation of collagen for depressed scars. J. Dermatol. Surg. Oncol. 6:450-453, 1980.

3. Adams, D. O. The biology of the granuloma. In: loachin, $\mathrm{H}$. (ed.), The Pathology of Granulomas. New York, Raven Press, 1980.

4. Boros, D. L. Granulomatous inflammation. Prog. Allergy 24:183267, 1978. 